\title{
CARACTERÍSTICAS DE LA PRÁCTICA PEDAGÓGICA DEL DOCENTE DE CONTADURÍA
PÚBLICA EN TIEMPOS DE NO PRESENCIALIDAD PEDAGÓGICA DEL DOCENTE DE CONTADURÍA
PÚBLICA EN TIEMPOS DE NO PRESENCIALIDAD CHARACTERISTICS OF THE PEDAGOGICAL PRACTICE OF PUBLIC ACCOUNTANCY TEACHERS DURING NON-CLASSROOM HOURS PEDAGOGICAL PRACTICE OF
}

\author{
Yair Rolando Casadiego Duque ${ }^{1}$ \\ Carlos Fabian Rico Rojas ${ }^{2}$
}

Raúl Prada Núñez ${ }^{3}$ 
los deberes académicos y familiares de forma simultánea. Por lo mencionado es que este trabajo reporta las características de la práctica docente y la posible influencia de los diversos recursos TIC bajo metodología no presencial en tiempos de aislamiento social en un programa de formación profesional de futuros Contadores Públicos en una universidad pública del nororiente colombiano. Se adopta un enfoque de investigación cuantitativo a nivel descriptiva transversal con diseño de campo. Se propone una encuesta ad hoc que satisfaga el objetivo perseguido, la cual se aplicó a una muestra aleatoria de 1006 estudiantes distribuidos entre el segundo y noveno semestre de su malla curricular. Los resultados permiten concluir que a pesar de llevar dos semestres en esta modalidad de trabajo aún existen limitaciones pedagógicas y aptitudinales en los docentes, lo cual impide garantizar un proceso de enseñanza pertinente.

\section{PALABRAS CLAVE:}

Educación superior, Práctica docente, recursos TIC, modalidad no presencial.

\section{SUMMARY}

This research takes place in the particular context, two semesters after the effects of the Covid-19 virus were recognised as a pandemic and compulsory social isolation was decreed in Colombia as a preventive measure. This situation affected all daily activities and especially the teaching paradigm rooted in the professional training processes, since it was necessary to abruptly migrate from a traditional face-to-face teaching model to a non-presential model assisted by technological resources. This has created a challenge for both students and teachers, who apart from dealing with all the difficulties derived from social isolation, must face the difficulties associated with this teaching approach, such as the lack of technological resources that guarantee access to activities both synchronously and asynchronously, the lack of qualifications in the use of these resources, and the interaction between academic and family duties at the same time. This paper reports on the characteristics of the teaching practice and the possible influence of the different ICT resources under non-presential methodology in times of social isolation in a professional training programme for future CPAs in a public university in north-eastern Colombia. A quantitative research approach is adopted at a cross-sectional descriptive level with a field design. An ad hoc survey is proposed to satisfy the objective pursued, which was applied to a random sample of 1006 students distributed between the second and ninth semester of their curriculum. The results allow us to conclude that in spite of having been in this modality of work for two semesters, there are still pedagogical and aptitudinal limitations in the teachers, which prevents us from guaranteeing a pertinent teaching process

\section{KEYWORDS:}

Higher education, teaching practice, ICT resources, distance learning.

\section{INTRODUCCIÓN}

Las instituciones de educación superior se enfrentan a grandes desafíos para la excelencia y la calidad en sus procesos de formación, siendo esto referentes importantes e impulsores en la búsqueda de cambios y transformaciones significativas que conlleven al desarrollo de la calidad en la enseñanza y aprendizaje demanda por la sociedad, de tal forma que logre aportar al progreso de los países. Fernández (2017) menciona que:

La educación superior es un bien de carácter estratégico para las naciones, como vínculo indisoluble entre la generación de capital humano altamente capacitado, la producción y difusión de conocimientos que favorecen 
la conformación de sociedades más justas y economías más competitivas. (p.184)

Sin embargo, Salas (2000) declara que:

Uno de los retos que se han planteado en muchos países en los últimos años representa la calidad de la formación y superación de los recursos humanos. Este proceso ha estado vinculado directamente a los cambios políticos, económicos y sociales que se han generado en los diferentes países, donde el desarrollo social, de la ciencia, la técnica, la práctica y la investigación, han obligado a aplicar no en el discurso, sino en la práctica efectiva los conceptos de eficiencia, calidad y exigencia en los procesos educativos que realizan las universidades, cada vez más comprometidas y en interacción con la sociedad. (p. 137)

En este sentido el mismo autor considera que la modernización de la Universidad enfrenta 4 retos fundamentales como son: "a) Equidad, calidad y eficiencia. b) Flexibilidad curricular, pertinencia social e investigación profesoral y estudiantil. c) Inserción, interacción y compromiso social de la universidad con el país. d) Modernización y descentralización de los procesos académicosadministrativos" (Salas, 2000, p.138).

Mientras que Urrego citado por Gómez (2012) indica:

La educación como función social atiende a la formación de sujetos que aporten al desarrollo social, por lo tanto, no sólo la educación universitaria tiene el compromiso de formar personas, sino que todo el sistema educativo debe aportar a la construcción de sujetos sociales. (p. 59)

Es por ello que requiere del compromiso de las instituciones de educación superior y los miembros de la comunidad educativa, fortalecer la formación permanente del docente y su aprendizaje continuo, acoplado a las necesidades y particularidades de los estudiantes, la gestión y administración curricular de alta calidad, la optimización de los procesos acreditación académica de las universidades, en el que permitan dar respuesta a las tendencias económicas, avances en la disciplina y contribuir en la solución de problemáticas sociales, organizaciones y empresariales.

\section{EDUCACIÓNSUPERIOREN LATINOAMÉRICA}

La Ciencia y la Tecnología se han convertido en uno de los principales pilares del desarrollo de la sociedad moderna. Su importancia ha aumentado exponencialmente a lo largo de los últimos siglos $\mathrm{y}$, en particular, en las últimas décadas. Esto es el resultado de la aparición de un nuevo paradigma tecno-económico basado en la producción circulación y transformación del conocimiento (Cajiao, 2019; Gamboa-Suárez, 2016).

El último informe del Observatorio 468 Iberoamericano de la Ciencia, la Tecnología y la Sociedad (OCTS-OEI) sobre Educación Superior publicado en marzo de 2019 señala varios procesos y rasgos que caracterizan la situación actual de las universidades en América Latina. En primer lugar, se destaca las transformaciones económicas y demográficas que han afectado la trayectoria de los sistemas nacionales de educación superior durante la última década. Por un lado, el boom de las materias primas iniciado alrededor de 2008 permitió la expansión y mejora de los sistemas universitarios en toda la región. Sin embargo, la crisis económica instalada desde 2015 trajo consigo recortes presupuestarios que frenaron o eventualmente detener este proceso. Por otro lado, la transición demográfica comienza a mostrar sus efectos a largo plazo en los sistemas educativos, frenando la demanda en los países más "viejos" (Brasil, Chile, Costa Rica o Cuba) o aumentando la presión sobre el sistema en 
los más "jóvenes" (Ecuador, Bolivia, México o El Salvador) (OCTS - OEI, 2019).

Las interacciones Universidad-Sociedad son una de las principales preocupaciones en el contexto latinoamericano. Durante las últimas décadas, las universidades han fortalecido sus capacidades científicas y tecnológicas pero estos logros no parecen transformar las condiciones económicas y sociales de la región, Para remodelar los sistemas de educación superior con el fin de crear y reforzar las interacciones Universidad-Sociedad y cumplir con la llamada "tercera misión" de la Universidad se ha convertido en un tema central en las agendas de las políticas públicas de los países latinoamericanos, el primer paso en esa dirección es evaluar el estado de estas interacciones y comparar diferentes experiencias en la región y en el extranjero (García, 2008; Bigi, García Romero y Chacón, 2018). Sin embargo, este primer paso no es fácil. La diversidad de interacciones que pueden darse entre estos dos ámbitos convierte esta tarea en algo muy complejo. Para hacer frente a esta complejidad, varios investigadores e instituciones a nivel local y regional llevan más de una década tratando de definir un marco común para medir estas interacciones.

Es difícil exagerar la importancia de la educación para el desarrollo económico y social de un país. Además de ser reconocida como un derecho humano fundamental Derechos Humanos, la Declaración de los Derechos del Niño (1959) y numerosas constituciones y documentos de gobierno de todo el mundo, una población bien educada garantiza el crecimiento sostenido y la prosperidad (Hanushek et al., 2008).

\section{RETOS Y AMENAZAS DE LA EDUCACIÓN SUPERIOR EN EL SIGLO XXI EN LATINOAMÉRICA}

De acuerdo con Arocena y Sutz (2005): ...a principios de la década de 1980 , las universidades estaban restringidas a las élites en los países latinoamericanos, con menos de 5 millones de estudiantes matriculados en sus cursos. Sin embargo, en las últimas cuatro décadas, la región atravesó un auge de instituciones de educación superior y ahora tiene más de 25 millones de estudiantes matriculados en más de 3.800 universidades, aproximadamente el $10 \%$ de todos los estudiantes matriculados en cursos de educación superior en el mundo con cuatro veces más instituciones de educación superior que Europa. (p.3)

La globalización ha transformado radicalmente el mundo en todos los aspectos. Pero ha transformado especialmente la economía mundial, que se ha vuelto cada vez más interconectada e interdependiente. Pero también hizo que la economía mundial fuera cada vez más competitiva y basada en el conocimiento, especialmente en los países occidentales desarrollados (Resta, 2004).

La educación global interconecta los métodos de enseñanza de los sistemas mundiales para fomentar el desarrollo internacional de la sostenibilidad ambiental, así como la contribución al fortalecimiento de las industrias globales. Estas iniciativas educativas priorizan el acceso global a la escuela desde el nivel primario hasta el universitario, promoviendo experiencias de aprendizaje que preparan a los estudiantes para roles de liderazgo multinacionales (Cepal, 2016, p. 12).

\section{EL EJERCICIO DOCENTE EN EL SIGLO XXI}

La tecnología, considerada como el nuevo lenguaje de la enseñanza y el aprendizaje, es una realidad irrevocable. Se espera que cualquier institución de educación superior mire hacia el futuro sin perder de vista sus capacidades centrales: la maleabilidad de sus docentes. Hoy, más que nunca, se espera que los docentes 
operen en un entorno donde predomine la alta tecnología (Hernández, 2017; López-García y Gutiérrez-Niño, 2018).

Las Tecnologías de la Información y la Comunicación - TIC mejoran el método de enseñanza-aprendizaje. Tal como, lo expresa la UNESCO citado por Carrera y Coiduras (2012) donde considera que:

La capacitación del docente en la utilización de las TIC resulta determinante para que estudiantes, trabajadores y ciudadanos sean capaces de: ( $1 \mathrm{r}$ enfoque) comprender las tecnologías (TIC); ( $2^{\circ}$ enfoque) resolver problemas complejos y prioritarios con los que se encuentran en situaciones reales en el trabajo, la sociedad y la vida; y (3r enfoque) comprometerse con la tarea de generar conocimiento, innovar y aprender a lo largo de toda la vida beneficiándose así tanto de la creación de este conocimiento como de la innovación y del aprendizaje permanente. ( $p$. 280)

Asimismo, es importante que los profesores cuenten con competencias digitales y a su vez sean incorporadas en sus actividades de enseñanza y aprendizaje, en razón a los cambios que se han venido configurando los procesos de formación, tal como lo establece Carrera y Coiduras, (2012) como son: "La adopción de recursos tecnológicos, vinculados al internet, tanto en la actividad desplegada en el aula como el trabajo autónomo del estudiante, y la utilización de entornos virtuales de enseñanzaaprendizaje como herramienta de apoyo a la docencia" (p. 278).

Por otra parte, para Tejada (2009) es importante considerar las competencias digitales como competencias inmersas en los docentes y en las cuales "se tendrá que abogar por el dominio de nuevas competencias profesionales que garanticen tanto el saber, como el saber hacer, el saber estar y el saber hacer en y con TIC" (p.12).

Es por ello que, en la práctica pedagógica del docente, el uso de las tecnologías cobran gran significancia en los procesos de enseñanza, en el que se deben precisar metodologías activas de manera que se logre mayor apropiación del recurso tecnológico y fortalecimiento de los procesos didácticos en la formación, el cual permita aportar ventajas para mejorar la eficiencia y aumentar la productividad en el aula, como también lograr incentivar el interés en los estudiantes en el desarrollo de sus labores académicas, aprovechando la red y sus potencialidades dentro del aula como fuera de ella.

Hay varios expertos de todo el mundo que han proclamado la importancia de las TIC en el proceso de enseñanza y se ha tenido en cuenta la revisión de la literatura para corroborar el papel de las TIC en la educación. Unos pocos de los miembros académicos han expuesto sus puntos de vista sobre la enseñanza de las TIC en la educación. "Las TIC no son sólo el futuro de la educación de nuestros hijos es el presente; y tenemos que invertir en las TIC ahora" (García et al., 2017; Martín, Hernández-Suárez y Mendoza-Lizcano, 2017).

Los valores, los talentos, las habilidades, el uso de la tecnología, la orientación, las habilidades necesarias, la empleabilidad junto con la credibilidad y la honestidad, acompañados de la búsqueda del conocimiento y el enfoque respectivo deben ser inculcados eficientemente en los estudiantes por medio de las TIC para que las utilicen en su futuro (Organización de las Naciones Unidas para la Educación, la Ciencia y la Cultura - UNESCO, 1998). Si la valiosa inversión se hace ahora mismo, los resultados serán extraordinarios para la mejora de las competencias digitales tanto en docentes como en estudiantes. 
Según McKenzie y Turner citado por Boobalakrishnan y Pichandy (2016), "Las TIC en la educación son la clave para desbloquear las habilidades y los conocimientos de nuestras futuras generaciones de jóvenes. Es la herramienta para el aprendizaje del siglo XXl". Además, el mismo autor menciona que "Las generaciones futuras nos agradecerán la lucha que estamos para promover las TIC y garantizar que tengamos una mano de obra de clase mundial que pueda competir en el mundo digital" (p. 377).

Es por ello que, el manejo constante de la tecnología constituye una base para el perfeccionamiento en el proceso de enseñanzaaprendizaje, proporcionando a las futuras generaciones habilidades competitivas en un mundo digital que cada vez es más cambiante.

Casi, la mayoría de los países occidentales utilizan las TIC desde la década de 1950 en el proceso de enseñanza, esto ha generado cambios en el rol docente y nuevos retos profesionales, requiriéndoles competencias digitales para llevar a cabo sus procesos formativos y ajustes a sus prácticas pedagógicas, en el que se destaca la necesidad de mitigar el impacto de la irrupción tecnológica, tal como lo plantean Lázaro et al. (2018), que a través de la "Generalización del uso de las TIC en el contexto actual ha producido cambios en el rol del profesorado (de trasmisor de conocimientos a guía del aprendizaje y del proceso la construcción del conocimiento" (p. 2). Por tanto, se hace inevitable la integración de las tecnologías de la información y la comunicación en los procesos de generación, trasferencia y preservación del conocimiento, en pro de una apuesta que posibilite al aprendizaje, la formación continua y permanente, que permita cubrir las necesidades educativas y de la sociedad.
Es un gran esfuerzo por parte de la actual generación de docentes para elevar el nivel educativo a un estatus de clase mundial. En este mundo digital modernizado, la adquisición de las competencias digitales es un gran reto, un desafío en todas las actividades del desempeño humano. Así que la gente se esfuerza por ser superior a los demás y también por elevar el nivel del país con respecto a otras naciones desarrolladas. Las TIC son una de las formas de elevar el nivel de la nación. De ahí que, trabajos como el de Rovira y Stumpo (2013) destacan la importancia del crecimiento infraestructura tecnológica promovida por el estado y el sector empresarial, provocan cambios cualitativos y cuantitativos en las económica y la sociedad. Además, afirman que "La utilización de estas tecnologías representa una oportunidad para generar beneficios que pueden aportar de manera significativa crecimiento económico, sino también a los procesos de inclusión social a través de mejores servicios en educación, salud y gestión gubernamental" (p.17). Así que el trabajo y los esfuerzos invertidos en el presente serán las generaciones futuras. Es la plataforma para que los jóvenes logren un éxito de clase mundial en el campo deseado.

La gran ventaja de utilizar las TIC es que disponen de diversas herramientas para enseñar a los estudiantes, tales como los portafolios electrónicos, quienes constituyen el método básico de enseñanza a través de las TIC, tal como se menciona:

La web es la fuente de toda la pedagogía de la enseñanza y el aprendizaje. Los registros de aprendizaje están estrechamente relacionados con el plan de aprendizaje, una herramienta emergente que se está utilizando para gestionar aprendizaje por parte de individuos, equipos, comunidades de interés y organizaciones. (Weldon et al., 2013, p. 45) 
Los portafolios electrónicos, al igual que los tradicionales, pueden facilitar la reflexión de los estudiantes sobre su propio aprendizaje, lo que lleva a una mayor conciencia de las estrategias y necesidades de aprendizaje.

Las iniciativas en materia de TIC de cualquier institución deben funcionar con objetivos de desarrollar, catalizar, apoyar y mantener la infraestructura y las actividades relacionadas con las TIC con el fin de mejorar el acceso, la calidad y la competencia en el sistema (Resta, 2004).

Las TIC pueden ser consideradas como un medio muy eficaz y eficiente para el proceso de enseñanza-aprendizaje (Vega, 2005). No sólo facilita un buen desempeño de los estudiantes, sino que es el mejor método de enseñanza para los profesores que les permite aprender cosas nuevas. El efecto de un mejor proceso de enseñanza-aprendizaje se traduce en la sucesiva de la nación.

\section{CAMBIOS DE PARADIGMAS DE ENSEÑANZA EN TIEMPOS DE CRISIS}

Gran parte de las medidas optadas por los gobiernos para afrontar la pandemia por el coronavirus (Covid-19) ha sido el cierre de actividades presenciales en las instituciones de educativas, como medida preventiva de propagación y mitigación de contagio, en el que la no presencialidad ha generado descontento social por limitantes socioeconómicas y las desigualdades tecnológicas, incidiendo en el rendimiento de los estudiantes. Umar, citado por Vertiz et al. (2019) declaran que "es inevitable señalar al estrés como una causa/consecuencia del fracaso académico de muchos estudiantes alrededor del mundo". (p.274). De ahí que, los cambios de paradigmas en la enseñanza, como consecuencia de la pandemia, han generado aumento en los estresores académicos, por lo que se venía trabajando bajo el modelo de enseñanza tradicional, cobrando mayor relevancia, ocasionando reacciones no esperadas, por las mismas exigencias de las instituciones de educativas, agudizando el rendimiento académico en los estudiantes. Por otro parte, el profesorado no ha sido ajeno a experimentar sentimientos de tensión y estrés, por el aumento de la carga laboral y las enseñanzas desde el hogar por el uso de las tecnologías. Sin embargo, Vertiz et al. (2019) afirman que:

En el contexto evolutivo, los docentes tendrían que adaptarse a los cambios generacionales, teniendo la responsabilidad de formar nuevos profesionales, competentes y globales, pero se hace necesario cambiar el paradigma de la educación centrado en el estudiante, pensando que también se hace imprescindible su formación sabiendo que, en el futuro, ellos serán los docentes de las generaciones venideras. (p. 281)

Por otra parte, Tello, citado por Flores y Navarrete (2020) menciona que se "deberá transitar del paradigma tradicional a la digital, a partir de actualización y capacitación a los docentes, propiciando la creación de una cultura tecnológica a partir de una educación centrada en las tecnologías... (p. 6)

Es por ello que, el aislamiento social impuso nuevos desafíos en la enseñanza-aprendizaje, por lo que hizo necesario abordar nuevas alternativas de solución no presenciales, necesarias para la capacitación docente en el desarrollo de estas habilidades tecnológicas para poder ejercer la labor docente. No obstante, Garcés et al. (2016) consideran que la tecnología aplicada a la educación supone una transición en los paradigmas educacionales, logrando la generación y exposición de los estudiantes a gran variedad de situaciones, actividades y experiencias formativas para lograr una mayor efectividad en su aprendizaje. 
Por lo que Toledo (2013), citado por Fernández et al. (2018) mencionan que las TIC:

En concreto pueden facilitar el acceso a todo tipo de información de manera más fácil y cómoda; favorecer la autonomía de los estudiantes, pudiéndose adaptar a las necesidades y demandas de cada alumno o alumna de forma personalizada; favorecer la comunicación sincrónica y asincrónica de estos estudiantes con el resto de compañeros y el profesorado; ayudan a la adaptación del entorno; mejoran el desarrollo cognitivo gracias a las actividades que sí que se pueden hacer; ahorrar tiempo para la adquisición de habilidades y capacidades; ... (p. 8).

Por consiguiente, la pandemia ha traído nuevos desafíos a nivel mundial, como cambios sociales, diseño de políticas educativas más incluyente, reformular los métodos de enseñanzaaprendizaje, mayor infraestructura y cobertura de acceso tecnológico, que permitan robustecer las capacidades tecnológicas de las instituciones educativas, el estado, los sectores productivo y la sociedad en general, logrando un mejor uso y aprovechamiento de las TIC, de tal forma que incidan en la generación de conocimiento y una mayor oportunidad competitividad.

\section{METODOLOGÍA}

Para el desarrollo de esta investigación se adoptó un enfoque cuantitativo, a nivel descriptivo transversal puesto que los datos son recolectados en los meses de abril y mayo del 2021, período de tiempo que corresponde al transcurso de doce meses después de haberse decretado el aislamiento social y por ende el cambio de metodología de enseñanza, tendiente a mitigar las afectaciones en los procesos de enseñanza, independientemente del nivel educativo en que se encuentren los estudiantes.
Se diseña un instrumento ad hoc en línea en el que se consideran dos secciones de preguntas con opciones de respuesta cerradas. Se inicia con el perfil demográfico de los estudiantes y posteriormente por medio de seis subsecciones, se explora en ellos la percepción que poseen actualmente sobre el desarrollo de procesos educativos en modalidad no presencial asistida por recursos tecnológicos, para luego evaluar la utilización por parte de los docentes de los recursos TIC en el desarrollo de las clases, el impacto que estas prácticas han tenido en su proceso de formación, para finalizar con el nivel de uso y recursos incorporados desde la plataforma institucional - PLAD. El instrumento es validado mediante dos mecanismos, inicialmente por el grupo de investigadores y el director del programa académico quienes actúan como panel de expertos para realizar la validez de contenido, posteriormente y tras la aplicación del instrumento a los informantes se determina el coeficiente Alfa de Cronbach, dada que en 68 ítems se aplica una escala Likert a cinco niveles. Los resultados derivados del coeficiente Alfa de Cronbach se muestran en la tabla 1 y permiten validar la fiabilidad del instrumento diseñado dado que el valor es de 0.958 que según Montgomery y Runger (2010) valores superiores a 0.80 son deseables. De forma complementaria, se determina la pertinencia de cada uno de los ítems considerados en el instrumento mediante el estadístico de total de elementos, en dónde se pudo verificar que la correlación total de elementos corregida arrojó en todos los casos un valor superior a 0.40 que según Muñiz et al. (2005) es lo deseable tal como se resalta en Prada et al. (2021), lo que permite garantizar la pertinencia de cada ítem dentro de la escala, conclusión que se ratifica al observar que si se suprime alguno de los ítems de la escala el valor del coeficiente Alfa no supera el ya reportado en la tabla 1. 
Tabla 1. Estadísticas de fiabilidad

\begin{tabular}{|c|c|}
\hline Alfa de Cronbach & N de elementos \\
\hline .958 & 68 \\
\hline
\end{tabular}

La población la conforman la totalidad de estudiantes matriculados para el primer semestre del 2021 en el programa académico de Contaduría Pública de una universidad del nororiente colombiano de naturaleza pública, totalizando 1566 estudiantes. Se intenta adelantar un estudio censal por ende no hay proceso de selección de muestra. A cada estudiante se le comparte el enlace del formulario y se le invita a diligenciarlo, obteniendo respuesta de 1006 estudiantes que representan el $64.2 \%$ de la población, por lo que se concluye que al final se aplicó un muestreo no probabilístico voluntario con dos criterios de inclusión: estar matriculado en el programa en el año 2021 y tener la disponibilidad para responder el instrumento.

Durante tres semanas se habilitó el diligenciamiento del instrumento, luego los datos fueron descargados del Google Form en un archivo de Excel para posteriormente ser exportados al software SPSS v25 con el fin de ser procesados de forma descriptiva.

\section{RESULTADOS Y DISCUSIÓN}

En esta sección se presentarán inicialmente los resultados para posteriormente generar el contraste de los resultados con trabajos previos a forma de discusión. Los resultados se presentan en función de la estructura del instrumento.

\section{SECCIÓN 1. PERFIL DEMOGRÁFICO DE LOS ENCUESTADOS}

Las características de los estudiantes encuestados son las siguientes: a) El $67.4 \%$ son mujeres, mientras que el porcentaje restante son hombres. El $92.2 \%$ provienen de los estratos 1 y 2 por lo que confirma la pertinencia del funcionamiento de la universidad en la ciudad a partir de su naturaleza pública dado que ofrece costes educativos accesibles.

En cuanto a la edad, se determinó que el $26.9 \%$ son menores de 20 años, mientas que el $63.9 \%$ se concentran en el rango de 20 a 30 años. El $70.4 \%$ son solteros y el $27.1 \%$ aseguran tener una relación estable bien sea mediante el vínculo del matrimonio (22.1\%) o por unión libre $(5.5 \%)$.

Finalmente, el $95.6 \%$ de los integrantes de la muestra aseguran que no poseen ningún tipo de discapacidad, entre tanto del porcentaje restante manifiestan que tienen limitaciones de movilidad o de visión.

\section{SECCIÓN 2. PERCEPCIÓN SOBRE EL DESARROLLO DE ACTIVIDADES ACADÉMICAS NO PRESENCIALES ASISTIDAS CON RECURSOS TIC}

Esta segunda sección del instrumento se ha dividido en seis subsecciones con número de ítems diferentes cada una de ellas y que se describe por medio de la Tabla 2.

Tabla 2. Subdimensiones de análisis de la percepción de los estudiantes sobre las actividades académicas actualmente desarrolladas

\begin{tabular}{|l|c|}
\hline \multicolumn{1}{|c|}{ Subdimensión } & $\mathbf{N}^{\circ}$ de ítems \\
\hline $\begin{array}{l}\text { Características de las clases no } \\
\text { presenciales }\end{array}$ & $\mathrm{P} 1-\mathrm{P} 9$ \\
\hline $\begin{array}{l}\text { Importancia actualmente de las } \\
\text { TIC }\end{array}$ & $\mathrm{P} 10-\mathrm{P} 15$ \\
\hline $\begin{array}{l}\text { Recursos que utiliza el docente } \\
\text { en su práctica pedagógica }\end{array}$ & $\mathrm{P} 16-\mathrm{P} 18$ \\
\hline $\begin{array}{l}\text { Medios educativos ofrecidos por } \\
\text { la Universidad }\end{array}$ & $\mathrm{P} 19$ \\
\hline $\begin{array}{l}\text { Proceso de retroalimentación } \\
\text { por parte del docente }\end{array}$ & $\mathrm{P} 20-\mathrm{P} 22$ \\
\hline $\begin{array}{l}\text { Recursos ofrecidos por la } \\
\text { plataforma institucional que el } \\
\text { docente utiliza }\end{array}$ & $\mathrm{P} 23$ \\
\hline
\end{tabular}




\section{CARACTERÍSTICAS DE LAS CLASES NO PRESENCIALES}

Respecto a esta subdimensión de análisis se pudo determinar que el $75.2 \%$ de los estudiantes aseguran que cuentan con las herramientas informáticas necesarias para el desarrollo de las clases no presenciales, mientras que el porcentaje restante manifiestan tener limitaciones en este sentido tal como se señala en la investigación de Avendaño et al. (2021), quienes resaltan las limitaciones de conectividad y de acceso a los recursos tecnológicos que poseen los estudiantes en tiempos de pandemia, puesto que en casa deben compartir el computador, smartphone o tablet con sus hermanos u otro familiar, al tiempo que se debe garantizar el acceso a internet permanentemente por lo que surge con un gasto más en el hogar (Lloyd, 2020).

Al explorar sobre el grado de satisfacción que tienen respecto a las clases no presenciales, el $45.2 \%$ manifiestan algún tipo de inconformismo que en algunos casos se han propiciado por las dificultades de accesibilidad, fallos en la red, falta de acompañamiento por parte de los docentes o simplemente, que les resulta más fácil distraerse en otras actividades (Díaz et al., 2020) mientras que el $37.6 \%$ asegurar estar satisfechos con ellas puesto que les ha permitido seguir estudiar a pesar de la pandemia.

Cuando se les preguntó a los estudiantes sobre, ¿cómo ha sido la enseñanza en las clases no presenciales? El $51.9 \%$ aseguran que han sido buenas, mientras que el $40.5 \%$ afirman que las clases no presenciales no promueven el aprendizaje dado que hay muchos aspectos por mejorar puesto que los docentes están intentando hacer por medio del computador las mismas actividades que se hacían de forma presencial, lo que se convierte en un ejemplo de la falta de cualificación de los docentes y de las posibles brechas existentes entre las instituciones de educación superior de naturaleza privada en las que ya venían invirtiendo recursos económicos y pedagógicos en metodologías de enseñanza no tradicionales tal como lo mencionan los trabajos de Lloyd (2020) y Kuric et al. (2021).

La falta de cualificación que se observa en los docentes en el uso de diversos recursos TIC, se contrasta con la opinión manifestada por el $66.9 \%$ de los encuestados quienes aseguran que ante esta situación de no presencialidad se han visto motivados a apropiarse en el manejo de los diversos recursos TIC, por lo que se convierte en un ejemplo de la brecha generacional que existe entre los actores del proceso educativo, en dónde los estudiantes son nativos digitales mientras que sus docentes podrían ser migrantes digitales (Cassany \& Ayala, 2008).

El hecho de estar estudiando desde casa ha evidenciado que este no es un espacio adecuado para esa actividad, dado que está expuesto a muchos distractores tal como lo reseña el $51.6 \%$ de los encuestados quienes aseguran que su concentración ha ido disminuyendo a medida que ha pasado el tiempo del aislamiento social, independientemente de que implemente nuevas formas de estudiar tal como lo señala el $91.6 \%$ de los estudiantes de la muestra.

Cuando se exploró sobre la herramienta informática más utilizada por los docentes en el desarrollo de las clases no presenciales, el $67.9 \%$ aseguran que ha sido la plataforma Google Meet, seguida de la plataforma institucional - PLAD en el $16.4 \%$ de los casos, situación que resulta coherente con lo mencionado en la investigación de Prada et al., (2019) en que los estudiantes reconocen la importancia del uso de la plataforma institucional pero resaltan como debilidad su escaso uso por parte de los docentes. La mensajería instantánea como el WhatsApp o el correo electrónico se presentan en el $4.4 \%$ y $2.1 \%$ de los casos, respectivamente. 
Finalmente, cuando se les pidió que evaluaran ¿cómo ha sido la comunicación con los docentes durante este tiempo? Se determinó que el $67.6 \%$ de los encuestados aseguran que ha mejorado dado que ahora el docente facilita el número del teléfono móvil, situación que anteriormente no era posible.

\section{IMPORTANCIA ACTUALMENTE DE LAS TIC}

Con esta subdimensión se pretendía caracterizar la percepción que poseen los estudiantes del programa de Contaduría Pública sobre el rol de los recursos TIC durante la enseñanza no presencial, por ello se indagó sobre si los docentes en el desarrollo de sus cursos han reforzado su práctica docente con el uso de recursos TIC, a lo que el $65.4 \%$ aseguran que sí por lo que resulta coherente con lo afirmado en los trabajos de Salinas (2004) y Palomo et al. (2001) en donde se enfatiza en la necesidad que tienen las instituciones de educación superior en la integración de las TIC en sus procesos de formación como estrategia de adaptación a las necesidades actuales de la sociedad; mientras que el $28.2 \%$ afirman que no es una práctica habitual o constante.

El $90.4 \%$ de los estudiantes encuestados reconocen que el uso de los recursos TIC mejora y enriquece el aprendizaje $y$ las competencias de los estudiantes, al tiempo que de forma recíproca lo hace con la práctica pedagógica de los docentes. En este sentido en el trabajo de Arancibia et al. (2020) se resalta que "en los profesores con predominancia constructivista, las concepciones sobre la relación entre aprendizaje y enseñanza ocupan un lugar central, con una preocupación mayor por crear entornos de aprendizaje significativo y de negociación, para el fomento y desarrollo del conocimiento" (p. 97).

Continuando con la sinergia y los beneficios que trae en el proceso de enseñanza, la combinación de recursos como lo son el trabajo colaborativo y el uso de los recursos TIC, se indagó sobre esta relación a los estudiantes, identificando que en el $62.9 \%$ de los casos aseguran que sus docentes han diseñado actividades de clase en las que interactúan estos dos recursos didácticos. En este sentido, "este enfoque también denominado co-constructivista se basa en el diálogo, en el aprendizaje colaborativo y en la aplicación del conocimiento a problemas auténticos con apoyo de la tecnología" (Arancibia et al., 2020, p. 97).

Como último aspecto en de interés en esta unidad de análisis, ha sido el pedirles a los estudiantes que valoren el desempeño de sus docentes en cuanto al uso de los recursos TIC en el desarrollo de las clases, a lo cual se identificó que el $70.7 \%$ consideran que ha sido adecuado y pertinente, mientras que el porcentaje restante de los estudiantes, consideran que sus docentes necesitan de procesos de cualificación en competencias tecnológicas.

\section{RECURSOS QUE UTILIZA EL DOCENTE EN SU PRÁCTICA PEDAGÓGICA}

Como se mencionó en la subdimensión anterior, hay docentes que parecen ser competentes en opinión de sus estudiantes, mientras que otros dejan en evidencia la necesidad de capacitación. En este sentido por medio de la Tabla 3 se puede identificar que los principales recursos utilizados por los docentes en el desarrollo de sus cursos, han sido las diapositivas y las guías de trabajo principalmente. En contraste con la utilización de películas, videos o mapas conceptuales como los menos utilizados. 
Tabla 3. Recursos metodológicos utilizados por los docentes.

\begin{tabular}{|l|c|c|c|}
\hline \multirow{2}{*}{\multicolumn{1}{c|}{ Recurso }} & \multicolumn{3}{c|}{ Escala valorativa } \\
\cline { 2 - 4 } & Nunca & Algunas veces & Siempre \\
\hline Guías & $18.0 \%$ & $27.6 \%$ & $54.4 \%$ \\
\hline Artículos & $27.4 \%$ & $34-6 \%$ & $37.9 \%$ \\
\hline Libros virtuales & $28.8 \%$ & $36.8 \%$ & $34.4 \%$ \\
\hline Bases de datos & $25.7 \%$ & $34.7 \%$ & $39.6 \%$ \\
\hline Películas y/o videos & $44.4 \%$ & $32.8 \%$ & $22.8 \%$ \\
\hline Diapositivas & $7.9 \%$ & $18.3 \%$ & $73.9 \%$ \\
\hline Mapas conceptuales & $33.1 \%$ & $28.8 \%$ & $38.1 \%$ \\
\hline Video conferencias & $12.5 \%$ & $16.2 \%$ & $71.3 \%$ \\
\hline Otros diferentes a los mencionados & $82.1 \%$ & $9.6 \%$ & $8.3 \%$ \\
\hline
\end{tabular}

Respecto al uso de estrategias docentes y métodos de enseñanza-aprendizaje, Montes y Machado (2011) afirman que las estrategias docentes se relaciona de forma dialéctica con los objetivos que persiguen, con la correcta selección del método de enseñanza, con los recursos didácticos que utilice y con las actividades que diseñe para alcanzarlos; todos articulados mediante la elaboración de un secuencia didáctica que debe ser ejecutada y controlada como parte del proceso de aprendizaje. Estos mismos autores aseguran que, "las estrategias docentes son válidas en su totalidad en un momento y un contexto específicos. La diferencia de grupos, estudiantes, profesores, materiales y contexto obliga a cada maestro a ser creador de estrategias docentes y métodos de enseñanzaaprendizaje" (p. 487).

El $83.6 \%$ de los estudiantes afirman que el material de apoyo a la clase siempre lo han recibido de forma oportuna, utilizando como principales canales de comunicación la plataforma institucional, el correo electrónico o la mensajería instantánea en el $91.9 \%, 71.8 \%$ y $64.8 \%$, respectivamente.

\section{MEDIOS EDUCATIVOS OFRECIDOS POR LA UNIVERSIDAD}

En esta subdimensión de análisis se intentan determinar el nivel de conocimiento y uso que tienen los miembros de la comunidad académica activa sobre los recursos que ha puesto la universidad a su disposición para el apoyo de los procesos de enseñanza y aprendizaje. En opinión de los estudiantes encuestados aseguran que el recurso más utilizado por ellos y sus docentes corresponde a las bases de datos disponibles desde la plataforma de la biblioteca con el $71 \%$ y $51 \%$, respectivamente. Le sigue en orden de prioridad en los estudiantes los E-books mientras que los docentes prefieren el libro escrito o impreso. 
Tabla 4. Medios educativos ofrecidos por la universidad para los estudiantes y docentes.

\begin{tabular}{|c|l|c|c|c|}
\hline \multirow{2}{*}{ Población } & \multirow{2}{*}{ Recurso } & \multicolumn{3}{|c|}{ Escala valorativa } \\
\cline { 2 - 5 } & Libros & Nunca & Algunas veces & Siempre \\
\cline { 2 - 5 } & Bases de datos & $25.9 \%$ & $39.3 \%$ & $34.8 \%$ \\
\cline { 2 - 5 } & E-books & $18.0 \%$ & $31.0 \%$ & $51.0 \%$ \\
\cline { 2 - 5 } & Ninguno & $24.8 \%$ & $31.1 \%$ & $44.1 \%$ \\
\hline \multirow{3}{*}{ Docentudiantes } & $54.7 \%$ & $15.6 \%$ & $13.1 \%$ \\
\hline & Libros & $12.9 \%$ & $25.8 \%$ & $61.1 \%$ \\
\cline { 2 - 5 } & Bases de datos & $9.5 \%$ & $19.5 \%$ & $71.0 \%$ \\
\cline { 2 - 5 } & E-books & $14.6 \%$ & $26.2 \%$ & $59.2 \%$ \\
\cline { 2 - 5 } & Ninguno & $72.5 \%$ & $14.3 \%$ & $13.3 \%$ \\
\hline
\end{tabular}

En lo que respecta a la preferencia a la incorporación de recursos TIC en el proceso académico en la Educación Superior, Calzada (2010) afirma que "la paulatina incorporación de las TIC a la docencia universitaria ... ha permitido pensar en un modelo de trabajo que precisamente busque la optimización de un valioso capital: los recursos didácticos" ( $p$. 315); ello como respuesta a las exigencias del mundo globalizado en el que se pretende alcanzar la excelencia por medio del máximo aprovechamiento de los recursos disponibles en la institución.

\section{PROCESO DE RETROALIMENTACIÓN POR PARTE DE LOS DOCENTES}

Esta unidad de análisis pretende determinar si en el proceso de enseñanza no presencial, los docentes realizan procesos de retroalimentación sobre cada una de las actividades que proponen a sus estudiantes. El $65.6 \%$ de los estudiantes encuestados afirman que sus docentes durante este año de pandemia siempre han realizado procesos de retroalimentación tanto de las actividades propuestas como de las evaluaciones aplicadas. Del porcentaje restante, el $27.1 \%$ aseguran que la retroalimentación sólo la realizan sobre los exámenes parciales, pero nunca sobre las actividades propuestas en el correr del curso.
Dentro de los recursos utilizados para realizar esta retroalimentación de actividades o también denominado asesorías, se identificó que el $56.1 \%$ utilizan el correo electrónico institucional, el $55.1 \%$ los foros de Meet Groups, el $50.1 \%$ el WhatsApp, el $70.2 \%$ prefieren hacerlo por medio de video conferencia de Google Meet o Zoom y el $80.8 \%$ lo realizan por medio de los diversos recursos de la plataforma institucional.

Al proceso de retroalimentación se le debe juzgar por la calidad del mismo, en este sentido el $69.1 \%$ de los estudiantes consultados manifiestan que durante estos espacios de reflexión académica han solventado sus dudas e inquietudes que se han derivado del proceso de aprendizaje. Respecto a la importancia del proceso de retroalimentación en el acto educativo, Gajardo (2019) afirma que "la retroalimentación se plantea como una intervención pedagógica, que se sustenta de información evaluativa y que brinda, tanto para docentes como para estudiantes, los pasos a seguir para continuar aprendiendo tanto de lo bueno como lo de malo" (p. 2).

\section{RECURSOS OFRECIDOS POR LA PLATAFORMA INSTITUCIONAL QUE EL DOCENTE UTILIZA}

Este análisis se finaliza con la identificación de los recursos didácticos que ofrece la plataforma institucional y que los docentes han incorporado 
durante estos doce meses de trabajo no presencial (ver Tabla 5). Se destacan como las actividades más populares entre los docentes organizados en orden de prioridad: talleres, tareas, foros, cuestionarios, uso de bases de datos y archivos adjuntos que complementan los temas desarrollados, con porcentajes de aceptación que oscilan entre $54 \%$ y el $77 \%$ de favorabilidad. En sentido contrario, las menos utilizadas son las wikis, las lecciones, los glosarios, las herramientas externas y las encuestas predefinidas.

Tabla 5. Recursos utilizados por los docentes y que están disponibles en la plataforma institucional.

\begin{tabular}{|l|c|c|c|}
\hline \multirow{2}{*}{\multicolumn{1}{c|}{ Recurso }} & \multicolumn{2}{c|}{ Escala valorativa } \\
\cline { 2 - 4 } & Nunca & Algunas veces & Siempre \\
\hline Bases de datos & $16.7 \%$ & $27.5 \%$ & $55.8 \%$ \\
\hline Chat & $24.8 \%$ & $28.6 \%$ & $46.5 \%$ \\
\hline Consultas & $15.5 \%$ & $26.3 \%$ & $58.1 \%$ \\
\hline Cuestionarios & $11.4 \%$ & $24.1 \%$ & $64.6 \%$ \\
\hline Encuestas predefinidas & $31.7 \%$ & $29.6 \%$ & $38.7 \%$ \\
\hline Foros & $9.0 \%$ & $25.6 \%$ & $65.4 \%$ \\
\hline Glosarios & $46.0 \%$ & $25.4 \%$ & $28.6 \%$ \\
\hline Herramientas externas & $35.2 \%$ & $30.2 \%$ & $34.6 \%$ \\
\hline Lecciones & $43.9 \%$ & $26.7 \%$ & $29.3 \%$ \\
\hline Talleres & $6.1 \%$ & $17.5 \%$ & $76.5 \%$ \\
\hline Tareas & $6.2 \%$ & $18.2 \%$ & $75.6 \%$ \\
\hline Wiki & $57.9 \%$ & $23.6 \%$ & $18.6 \%$ \\
\hline Archivos & $21.3 \%$ & $23.9 \%$ & $54.7 \%$ \\
\hline Libros & $30.1 \%$ & $27.7 \%$ & $42.2 \%$ \\
\hline Páginas web & $31.4 \%$ & $26.5 \%$ & $42.1 \%$ \\
\hline
\end{tabular}

\section{CONCLUSIONES}

Los docentes del programa académico de Contaduría Pública de una universidad pública del nororiente colombiano, han tenido aceptación en la incorporación de recursos TIC en el desarrollo de su práctica pedagógica, utilizando como herramienta pedagógica para sus clases las diapositivas, donde compilan los temas a tratar y hacer que los estudiantes encuentren allí conceptos y demás.

Tanto los docentes como los estudiantes reconocen la importancia que han tenido las bases de datos en estos meses de no presencialidad en el proceso de consulta académica, dado que proporcionan materiales que puedan facilitar el proceso educativo mediante el uso de las tecnologías existentes. Se destaca en este sentido, la diferencia existente entre los estudiantes y los docentes, donde los primeros prefieren consultar los libros digitales mientras que los docentes optan con mayor favoritismo por las versiones impresas de los mismos. Esto posiblemente puede estar propiciado por las facilidades de acceso a los recursos impresos que tienen los docentes en comparación con los estudiantes.

Los procesos académicos están acompañados de retroalimentación, está relacionada más fuerte y consistentemente con el rendimiento que cualquier otro comportamiento de enseñanza. Adicional de los encuentros sincrónicos los docentes recurren a la plataforma institucional 
para enviar material de apoyo a las clases, mientras que prefieren las sesiones por Google Meet como recurso de apoyo para realizar los procesos de retroalimentación con sus estudiantes.

Por último, las tecnologías han sido fundamental apoyo para el desarrollo docente, a pesar de que en opinión de los estudiantes y debido a que han pasado más de doce meses en esta situación, aún persisten docentes que no poseen las competencias tecnológicas necesarias para aprovechar tantos recursos disponibles, que sumado a un ejercicio de cualificación pedagógica en la que se instruyan en la correcta utilización de estos recursos con el fin de potenciar el proceso de enseñanza.

\section{REFERENCIAS BIBLIOGRÁFICAS}

Arancibia, M. L., Cabero, J. y Marín, V. (2020). Creencias sobre la enseñanza y uso de las tecnologías de la información y la comunicación (TIC) en docentes de educación superior. Formación universitaria, 13(3), 89-100.

Arocena, R. y Sutz, J. (2005). Latin American Universities: from an original revolution to an uncertain transition. Higher Education, 50(4), 613-630.

Avendaño, W. R., Gamboa, A. A. y Prada, R. (2021). Jaque a la educación: percepciones de maestros en formación de una universidad pública sobre la calidad de la enseñanza en tiempos de crisis. Revista Boletín Redipe, 10(5), 71-82. https://doi.org/10.36260/rbr. v10i5.1286

Bigi, E., García Romero, M., \& Chacón, E. (2018). Cultura institucional y su impacto en las prácticas de lectura y escritura en la universidad. Revista Perspectivas, 3(2), 46-59. https://doi. org/10.22463/25909215.1588
Fernández, J. M ${ }^{\mathrm{a}}$, Reyes, M. $\mathrm{M}^{\mathrm{a}}$ y El Homran, M. (2018). TIC y discapacidad. Principales barreras para la formación del profesorado. EDMETIC, Revista de Educación Mediática y TIC, 7(1), 1-25. https://doi.org/10.21071/edmetic. v7i1.9656

Boobalakrishnan, N., y Pichandy, C. (2016). ICT and higher education: Teachers technological driven ecosystem and a paradigm shift in learning environment. ICTACT Journal On Management Studies, 2(4), 377-383. https://doi. org/10.21917/ijms.2016.0049

Cajiao, F. (2019). Educación superior en América Latina y el Caribe: desafíos y asuntos pendientes. En Instituto Internacional de Unesco para la Educación Superior en América Latina y el Caribe, La Universidad del Siglo XXI en América Latina y El Caribe: un debate en desarrollo (pp. 161-180). Educación Superior y Sociedad. https://www.iesalc. unesco.org/ess/index.php/ess3/article/ view/54/55

Calzada, F. J. (2010). Repositorios, bibliotecas digitales y CRAl: los objetos de aprendizaje en la educación superior. Alfagrama Ediciones

Carrera, F. y Coiduras, J. (2012). Identificación de la competencia digital del profesor universitario: un estudio exploratorio en el ámbito de las Ciencias Sociales. REDU. Revista de Docencia Universitaria, 10(2), 273-298. https://doi. org/10.4995/redu.2012.6108

Cassany, D. y Ayala, G. (2008). Nativos e inmigrantes digitales en la escuela. Participación educativa: revista del Consejo Escolar del Estado, 9(4), 57-75. 
Cepal. (2016). Educación y Globalización_los desafíos para América Latina (1. ${ }^{a}$ ed.). CEPAL, OEI.

Díaz, D, Fernández, Heimann, A. G. y Ayala, N. L. (2020). Dificultades en la aplicación de clases remotas durante la pandemia de covid-19 en la FACITEC. Tecnología, Diseño e Innovación, 6(1), 1-17.

Fernández, E. (2017). Una mirada a los desafíos de la educación superior en México. Innovación educativa, 17(74), 183-207.

Flores, M. R. y Navarrete, C. A. (2020). Diagnóstico de necesidades de capacitación en el uso de plataformas virtuales ante la contingencia del COVID-19 en los estudiantes y docentes de Educación Media Superior Tecnológica. Dilemas contemporáneos: educación, política y valores, 8(spe17), 1-21. https://doi.org/10.46377/dilemas. v8i.2494

Gajardo, C. G. (2019). Falta de eficacia en la retroalimentación en el proceso evaluativo de los estudiantes [tesis doctoral, Universidad del Desarrollo]. Repositorio Institucional UDD. https:// repositorio.udd.cl/handle/11447/2830

Gamboa-Suárez, A. A. (2016). Docencia, investigación y gestión: Reflexiones sobre su papel en la calidad de la educación superior. Revista Perspectivas, 1(1), 81-90. https://doi. org/10.22463/25909215.973

Garcés, E., Garcés, E. y Alcívar, O. (2016). Las Tecnologías de la Información en el cambio de la Educación Superior en el siglo XXI: reflexiones para la práctica. Universidad y Sociedad, 8(4), 171-177.

García, M. J. (2008). El impacto de la globalización en la universidad del siglo
XXI. Tendencias Pedagógicas, 13, 5978.

García, M. del R., Reyes, J. y Godínez, G. (2018). Las Tic en la educación superior, innovaciones y retos / The ICT in higher education, innovations and challenges. RICSH Revista Iberoamericana De Las Ciencias Sociales y Humanísticas, 6(12), 299 - 316. https://doi.org/10.23913/ ricsh.v6i12.135

Gómez, Y. (2012). Educabilidad: una resignificación conceptual en contabilidad desde la educación crítica. Universidad de Antioquia, (61), 91-110

Hanushek, E. A., Jamison, D. T., Jamison, E. A. y Woessmann, L. (2008). Educación y crecimiento económico: no es solo ir a la escuela, sino aprender algo mientras está allí lo que importa. Educación siguiente, 8(2), 62-70.

Hernández, R. (2017). Impacto de las TIC en la educación: Retos y Perspectivas. Propósitos y Representaciones, 5(1), 325-347. http://doi.org/10.20511/ pyr2017.v5n1.149

Kuric, S., Calderón, D. y Sannmartín, A. (2021). Educación y brecha digital en tiempos del COVID-19. Perfiles y problemáticas experimentadas por el alumnado juvenil para continuar sus estudios durante el confinamiento. Revista de Sociología de la Educación-RASE, 14(1), 63-84.

Lázaro-Cantabrana, J. L., Gisbert-Cervera, M. y Silva-Quiroz, J. E. (2018). Una rúbrica para evaluar la competencia digital del profesor universitario en el contexto latinoamericano. Edutec. Revista Electrónica De Tecnología Educativa, (63), 1-14. https://doi.org/10.21556/ edutec.2018.63.1091 
Lloyd, M. W. (2020). Desigualdades educativas y la brecha digital en tiempos de COVID-19. En H. Casanova Cardiel (Coord.), Educación y pandemia: una visión académica (pp. 115-121). Universidad Nacional Autónoma de México, Instituto de Investigaciones sobre la Universidad y la Educación.

López-García, J. D., \& Gutiérrez-Niño, D. (2018). Efecto del uso de la herramienta "realidad aumentada" en el rendimiento académico de estudiantes de Educación Básica. Revista Perspectivas, 3(1), 6-12. https://doi.org/10.22463/25909215.1464

Martín, M. M., Hernández-Suarez, C. A., \& Mendoza-Lizcano, S. M. (2017). Ambientes de aprendizaje basados en herramientas web para el desarrollo de competencias TICenla docencia. Revista Perspectivas, 2(1), 97-104. https://doi. org/10.22463/25909215.1282

Montes, N. y Machado, E. F. (2011). Estrategias docentes y métodos de enseñanzaaprendizaje en la Educación Superior. Humanidades médicas, 11(3), 475-488.

Montgomery, D. C. y Runger, G. C. (2010). Applied Statistics and Probability for Engineers. John Wiley \& Sons.

Muñiz, J., Fidalgo, A. M., García, E., Martínez, R. y Moreno, R. (2005). Análisis de ítems. La Muralla.

Observatorio Iberoamericano de la Ciencia, la Tecnología y la Sociedad. (2019) ¿Es baja la inversión en I+D de los gobiernos iberoamericanos? http://www. ricyt.org/2019/08/informe-de-coyunturan-03-es-baja-la-inversion-en-id-de-losgobiernos-iberoamericanos/
Organización de las Naciones Unidas para la Educación, la Ciencia y la Cultura. (1998). Declaración munidal sobre la educación superior en el siglo XXI, Visión y acción. Educación Superior y Sociedad, 9(2), 97-113.

Palomo, R., Ruiz, J. y Sánchez, J. (2010). Enseñanza con TIC en el siglo XXI. La escuela 2.0. Educatio Siglo XXI, 28(2), 291-294.

Prada Núñez, R., Hernández Suárez, C. A. y Fernández-Cézar, R., (2021). Determinantes afectivos, procedimentales y pedagógicos del rendimiento académico en matemáticas. Aproximación a una escala de valoración. Revista Boletín Redipe, 10(3), 202-224. https://doi.org/10.36260/rbr.v10i3.1229.

Prada, R., Hernández, C. A. y Gamboa, A. A. (2019). Usos y efectos de la implementación de una plataforma digital en el proceso de enseñanza de futuros docentes en matemáticas. Revista Virtual Universidad Católica del Norte, (57), 137-156.

Resta, P. (2004). Las tecnologías de la información y la comunicación en la formación docente. Guía de planificación. Unesco.

Rovira, S. y Stumpo G. (2013). Entre mitos y realidades. TIC, políticas públicas $y$ desarrollo productivo en América Latina. Naciones Unidas. https:// repositorio.cepal.org/bitstream/ handle/11362/37248/1/LCL3600_es.pdf

Salas, R. S. (2000). La calidad en el desarrollo profesional: avances y desafíos. Educación Médica Superior, 14(2), 136147. 
Salinas, J. (2004). Innovación docente y uso de las TIC en la enseñanza universitaria. Revista Universidad y Sociedad del Conocimiento, 1(1), 1-16. http://doi. org/10.7238/rusc.v1i1.228.

Tejada, J. (2009). Competencias docentes. Profesorado. Revista de currrículum y formación del profesorado, 13(2), 1-15.

Toledo, P. (2013). Las tecnologías de la información, la comunicación y la inclusión educativa. En J. Barroso y J. Cabero. (Coords.), Nuevos escenarios digitales (pp. 411-426).Pirámide.

Vega, R. (2005). La educación continua a distancia en México: Transformaciones y retos. Revista de la Educación Superior, 34(133), 79-86.

Vértiz-Osores, J. J., Vílchez, G. L., VértizOsores, R. I., Damián-Núñez, E., Chico, H. y Rodríguez-Fuentes, A. (2019). Malestar docente: reflexiones frente al bajo rendimiento académico en estudiantes universitarios. Propósitos y Representaciones, 7(3), 273-299. http:// doi.org/10.20511/pyr2019.v7n3.387

Weldon, P., McKenzie, P., Kleinhenz, E. y Reid, K. (2013). Teach for Australia Pathway: Evaluation Report Phase 3 of 3. Australian Council for Educational Research. https:// research.acer.edu.au/cgi/viewcontent. cgi?article $=1013 \&$ context=teacher education 